\title{
Between territories: Incremental changes to the domestic urban interface between private and public domains
}

\author{
Agnieszka Wir-Konas, Kyung Wook Seo \\ Northumbria University, Newcastle upon Tyne. UK \\ E-mail: agnieszka.wir-konas@northumbria.ac.uk,kyung.seo@northumbria.ac.uk
}

\begin{abstract}
In this paper we investigate incremental changes to the relationship between private and public territory on the micro-morphological scale of the dwelling-street interface. This interface lies on the edge between two distinctively different spatial domains, the house and the street, and provides a buffer which may be adjusted to aid the transition from private to public territory. The structure of the space impacts both domains: it provides a fit transition from the private $d$ welling to the public territory, creates a space for probabilistic encounters between inhabitants and strangers, and maintains the liveability of the public street. The aim of this paper is threefold: Firstly, we recognise morphological differences in the structure of the interfaces and the way the transition from private to public territory was envisioned and designed in different societal periods. Secondly, we study incremental changes to the interface, representing individual adjustments to the private-public boundary, in order to recognise common types of adaptations to the existing structure of the interface. The history of changes to each individual building and building-street interface was traced by analysing planning applications and enforcements publicly provided by the city council. Lastly, we compare the capacity of each dwelling-street interface to accommodate incremental change to the public-private transition. We argue that studying the incremental change of the interface and the capacity of each interface to accommodate micro-scale transformations aids in the understanding of the complex social relationship between an individual and a collective in the urban environment.
\end{abstract}

Keywords: Urban morphology, micromorphology, urban interface, incremental change

\section{Introduction}

As established in urban morphology, urban form consists of elements - e.g. buildings, plots and streets (Conzen, 1960; Kropf, 2009) which apart from their own form and properties, establish a certain relationship with each other. The space, which is a result of this relationship can be defined as an urban interface, providing and maintaining the relationship between two different territories. In this paper, we discuss a specific case of an urban interface created as a result of a relationship between a house and a street, which we call the dwelling-street interface (following the naming convention introduced in Palaiologou et al., 2016). The concept of the dwelling-street interface was widely discussed as an important element for maintaining liveability in the street, maintaining important relationships between inhabitants and strangers and enabling individuals to exercise control over the privatepublic boundary and the way they transition between those two territories (Whitehand et al., 1999; Brown et al., 1998; Skjaeveland and Garling, 1997; Lawrence, 1987; Gehl, 1986; Jacobs, 1961, Palaiologou et al., 2016). Even though the importance of the urban interface is discussed there are very few morphological studies of the interface. 
In the book The Environment and Social Behavior: privacy, personal space, territory, crowding Altman argues that the balance between the private and public in our relationship with other people is in a constant flux (Altman, 1975) and is undergoing constant changes and adjustments to fit our current needs for privacy or social contact. In urban form, the frequent changes and shifts to the spatial interface are not always possible but different approaches to the concept of the relationship between the private house and public street are visible not only in a variety of interface forms from different morphological periods, but also in incremental changes initiated by inhabitants themselves. The comparison of the spatial interfaces designed by speculative developers and those altered by inhabitants creates a discussion on the gap between architects' understanding of the spatial relationship between the house and the street and inhabitants' personal outlook. The structure of this paper is as follows: Firstly, we provide a short historical overview of Gosforth, a district of Newcastle upon Tyne. Secondly, we discuss the morphology of the dwelling-street interface and describe the types of interfaces observed in the sampled area. Finally, we discuss incremental changes to the interface and provide an overview of the difference between designed dwelling-street interfaces and those incrementally adjusted by inhabitants. We conclude with a paragraph on the capacity of different types of interfaces to accommodate change.

\section{Case study and methodology - Gosforth}

The development of the urban form in Gosforth (a district of Newcastle upon Tyne, UK) is intertwined with the rise and fall of the mining industry. The demand to meet the accommodation needs of the working class miners and, after the decline of the mining industry, the emerging middle class led to the rapid expansion of speculative housing estates. The developing residential urban form can be divided between five morphological periods, each connected with speculative developments with varying housing typologies and different outlooks on the relationship between the street and a dwelling. The first speculative housing estates were built in the 1890s and were situated between the urban nucleus of Gosforth - Bulman Village - and the nearby Coxlodge Colliery. The estates were made up of terraced houses and Tyneside flats ${ }^{1}$ providing accommodation mostly to the mineworkers. Development of speculative estates prioritising accommodation for the growing mining workforce continued throughout the 1910s. Between the 1940s and the 1970s the mining industry began to decline and speculative developers shifted their focus to the emerging middle class, building new estates consisting predominantly of semi-detached houses. Since the 1990s the dominant housing typology in the district changed to detached housing and tree-like suburban estates are currently the most common speculative estate type.

To trace the similarities and differences between the structure of the dwellingstreet interface we collected information on the geometry of the interfaces based on historical plans and Ordnance Survey maps. To understand the logic of the relationship between the street and the house and discover underlying interface types, we studied plans and focused on geometry, layout and configuration of the convex spaces. The treatment of the relationship between the street and the dwelling, represented by each of the interface types from different morphological periods, might be disconnected from the way in which current inhabitants view this relationship. If the space is not fit to accommodate necessary needs, the inhabitants will most likely address the problem and incrementally adjust the configuration to suit their personal requirements. To track the changes in the geometry of the interfaces we studied planning applications and building permissions accessed through the Newcastle City Council's planning database ${ }^{2}$ and documented any change to each property in the sample. However, this method had a few limitations. Not all changes to the geometry require planning permission and/or building permission, as it depends on the dimensions of the proposed extension, location in relation to the house and distance to the boundary of the 
property. Moreover, the registry of applications in the Gosforth area only dates back to 1975 , which means information on changes to the geometry before that point is lost. In order to overcome those limitations, we documented changes in geometry based on a comparison between historic and the latest Ordnance Survey maps. To confirm data collected from both planning application and plan comparison we conducted an on-site observation study and documenting the changes to the interface.

\section{The morphology of the dwelling-street interface}

The dwelling-street interface is a spatial outcome of the relationship between a house and, through the adjacent street segment, a complex street network. Through the interface, the house and the street meet, interact with and affect each other. Being a spatial by-product of a connection between a house and a street, the dwelling-street interface is not an element on its own, as it would not exist without a house and a street; therefore it cannot be studied in isolation. The relationship between a dwelling and a street can be categorised based on the number of connections between a house and the street network. In the studied area we distinguished three general types, where a house is connected to the street network through: one, two or three street segments. The one house-one street relationship is common to the semi-detached and detached houses where

(a)

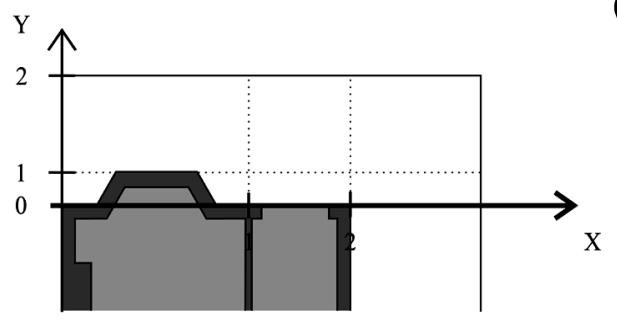

front façade is facing only one street segment. The one house-two streets relationship can be seen in terraced houses, where both front façade and back exterior wall are facing a street. Additionally, we can observe this relationship in corner semi-detached and detached houses. The one house-three streets relationship can be found in corner terraced houses, where three exterior walls of a house are facing different street segments. Based on this distinction and the types of exterior wall adjacent to the street segments, we distinguished three types of the interface: front, side and back. As discussed, the distribution of front, side and back interfaces varies between housing typologies. The front interface appears most consistently and is the most numerous out of all the types; therefore will be the focus of this paper. The study of the side and back interface while equally interesting will be addressed in future work.

The structure of the front interface can be divided between constant and variable elements. The two elements that are part of every front interface are the building exterior wall and plot boundary. They demarcate the edges of private domestic and public territories. The building exterior wall restricts access and visibility into the domestic territory, while the plot boundary controls the access but allows strangers to look inside. In the majority of cases studied in Gosforth (98.52 per cent of all houses) there is, additionally, a convex space (or set of convex spaces) introduced between the house and the street. In the paper we include this convex space between the building exterior wall and

(b)

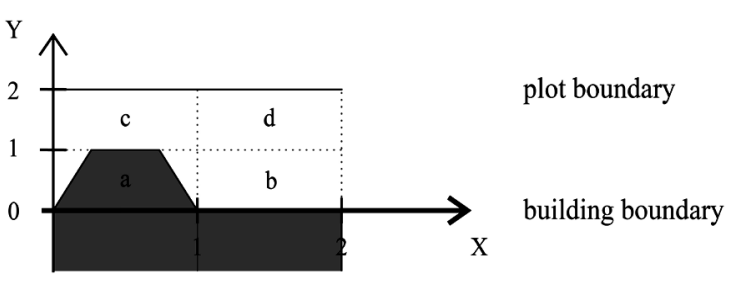

$$
2(1,0,0) \times 2(1,0,0) \times 2
$$

Figure 1.

(a) Plan of a front interface with grating marking convex spaces (b) 'Dimensionless representation' (Steadman, 1983) of the same plan. The type of the interface presented in the diagram is marked as $2(1,0,0) \times 2$ where, $2 \times 2$ describes number of convex spaces on the $X$ and $Y$ axes and the numbers in brackets show the number of bay windows, porches/alcoves and garages. This type of the interface is categorised as medium complexity. 
plot boundary as the third constant element. The varying elements can be divided into two groups: extensions of the domestic space into the semi-private area with e.g. a bay window or a porch; and extensions of the semi-private area into the domestic territory with e.g. alcoves. The relationship between those different elements creates a structure consisting of private convex spaces, extending the domestic territory to the outside and the semi-private convex spaces, which introduce the connection of public territory to the private property. The complexity of the interface structure varies with examples where the privacy-orientated territory dominates the interface, on the other hand, there are houses where this relationship is reverted and the semi-private space penetrates into the house. The number and types of convex spaces between the house and the street set the complexity of the connection between those two territories. The structure of the interface not only describes how complex the entry to the house is, but also the capability of the interface to accommodate activities which can generate a random encounter between the inhabitants and strangers. In British housing typologies the houses differ in their position on the plot, which, in the case of semi-detached and detached housing creates a space between the side of the building and the plot line. In determining the interface typologies we have not included the side space as an element because it unnecessarily enlarges the number of the types. The information on the position of the house on the plot was preserved and used further in the analysis of changes to the interface.

In the Gosforth area we observed a total of 51 types of front interfaces distributed between all three housing typologies. We presumed that each housing typology creates a unique interface with adjacent streets, but we found that the majority of interface types are shared across at least two housing typologies. The houses with interface types unique to the housing typology constitute 14 per cent of all houses, while the houses that share interface types among all three housing typologies constitute 59 per cent of all houses. In Gosforth there are 29 interface types unique to the housing typologies and 4 interface types that are shared across all typologies. This could suggest that the relationship between the house and the street is not connected to the housing typology, but only if we treat a housing typology as a building only, without considering its position on the plot.

Furthermore, we distinguished four categories of interface typology based on the complexity of the layout and the position of the exterior wall. The first category is the simplest example of the interface with the domestic territory ending on the exterior wall and not encroaching onto the semi-private area. In this case, there is one convex space separating the house and the street with one step between the public and private territory. The second category describes the interface where the domestic territory extends into the semi-private space with e.g. a bay window or a porch. This extension divides the convex space between the house and the street and creates an additional step between those territories. The third category introduces a reversed scenario, where the semi-private territory steps into the building as e.g. an alcove, and introduces an additional step past the exterior wall. Finally, the forth category, describes the types where the domestic territory expanded so greatly it created a new iteration of the exterior wall, one step closer to the street. All of the categories discussed above are present in all of the housing typologies. An overview of the most common front interface types divided between the four categories and main housing typologies is shown in Figure 2.

\section{Incremental changes to the dwelling-street interface}

The perception and treatment of the relationship between the private and public territory is shifting with the changing socio-cultural background. The dwellings and their interfaces built, for example, in the 1910s, refer to a wholly different cultural environment and some of the design decisions might not be applicable to current attitudes. In order to match the presentday perception of the relationship between the private and the public, the interface had to be incrementally adjusted by inhabitants. 
(a) terraced houses

$1 \longrightarrow$ plot boundary - edge between street and plot
building boundary - exterior wall adjacent to a street segment plot boundary - edge between street and plot $1(0,0,0) \times 1$

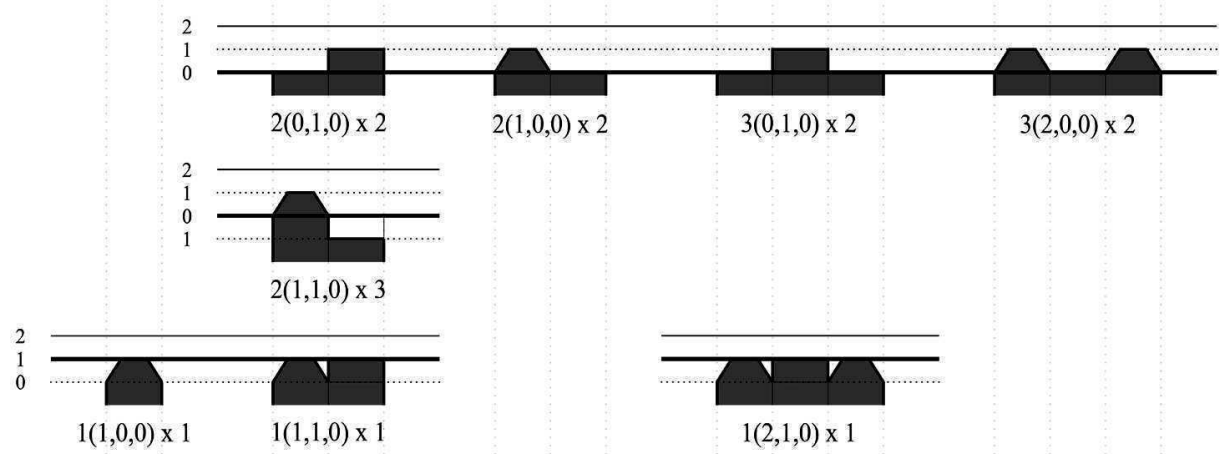

(b) semi-detached houses

1
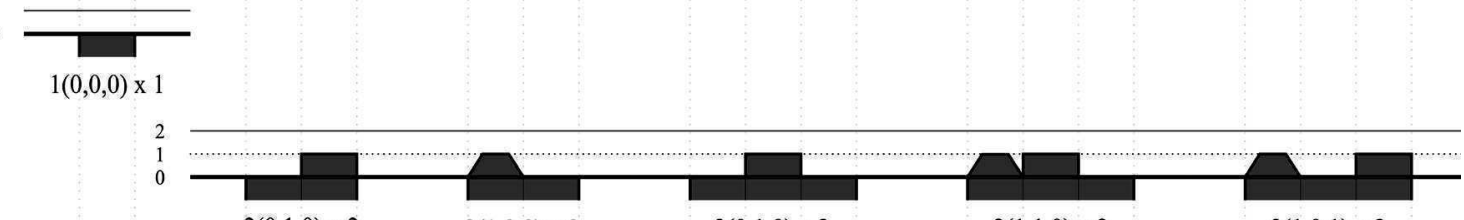

$2(0,1,0) \times 2 \quad 2(1,0,0) \times 2$

$3(0,1,0) \times 2$

$2(1,1,0) \times 2$

$3(1,0,1) \times 2$

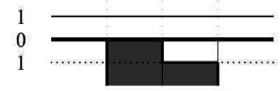

$2(0,1,0) \times 2$
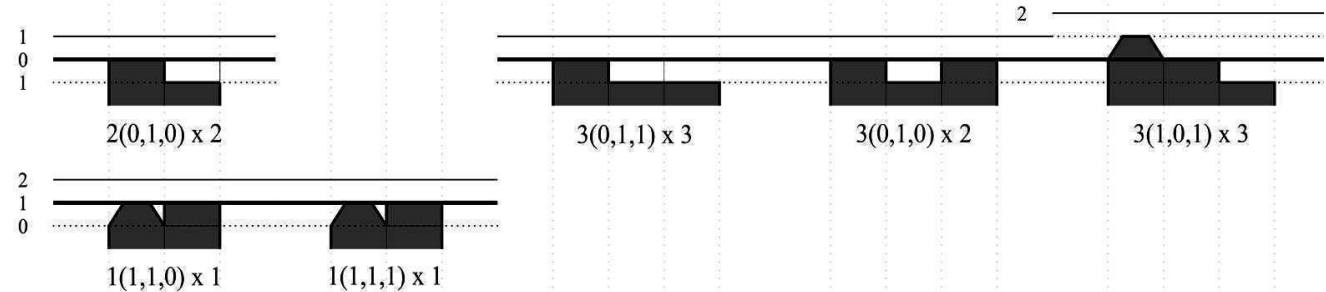

(c) detached houses

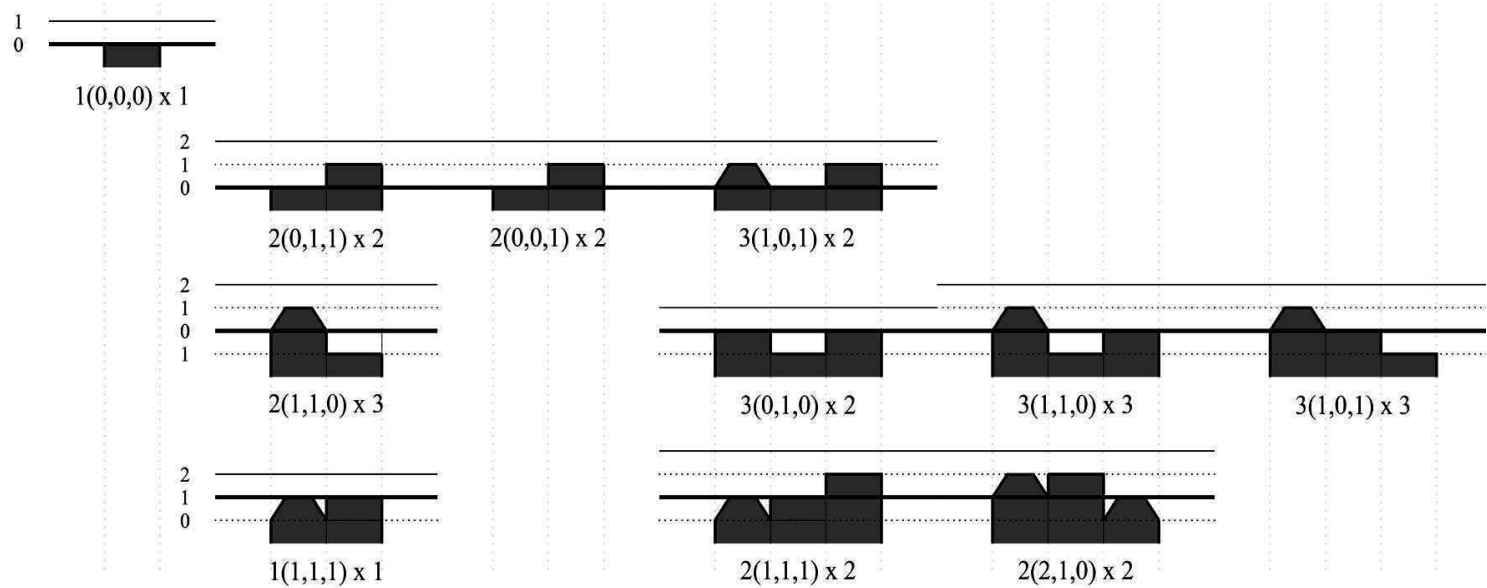

Total number of 16 in terraced, 36 in semi-detached and 28 in detached houses front interface types were found in Gosforth area.

Figure 2.

Atlas of the common front interface typologies. The solid space represents the private domestic territory, while the void space represents the semi-private space visible to the public from the adjacent street. 
The types, patterns and distribution of these changes are the main focus of this paragraph, which is followed by a discussion on the capacity to accommodate change amongst interfaces in different housing typologies. We distinguish three types of incremental changes applicable to the front interface: change that resulted in a lower complexity of the space between the house and the street, change that did not affect the complexity of the space and change that raised the complexity of the space. Additionally, we differentiate between addition and subtraction from the geometry. We are concerned only with changes to the geometry, therefore we did not include any changes to the access points in the analysis.

The overall number of changes to the front interface varies between the housing typologies, with 10 per cent of the terraced houses, 63 per cent of semi-detached and 36 per cent of detached houses being incrementally adjusted over time. In all housing typologies the majority of interface types changed at least once, showing that each interface type has the capability to accommodate change (see Table 1). However, the study showed that only 25 to 30 per cent of all interface types amongst all housing typologies underwent the majority of changes to the front interface. Although most interface types are capable of change, only a few types are flexible enough to accommodate different types of changes.

In terraced housing the original interface typologies can be divided between spaces with low complexity (e.g. 1 x 1), medium complexity (e.g. $2 \times 2$ ) and high complexity (e.g. $3 \times 2$ ). The complexity of the original interface type correlates with the type of change introduced by inhabitants. The low complexity types rarely changed but when the change was introduced it resulted in higher complexity of the interface. The interface types with medium complexity accommodated the largest variety of changes and led to both increasing and decreasing of the complexity of the interface. Finally, change to the interface types with high complexity resulted in a lowering of the complexity and a simplification of the access to the house in most cases. The majority of incremental changes were applied to the interface types with medium complexity.

The most popular change was a reduction of the porch, which depending on the interface type resulted in lower or higher complexity of (a)

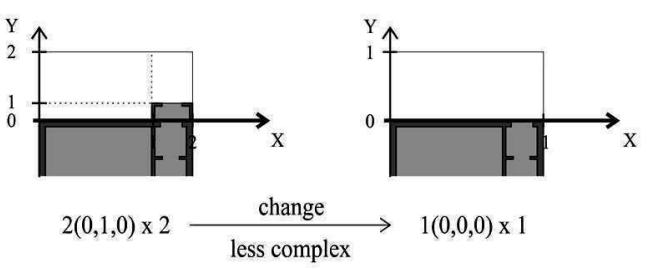

(b)

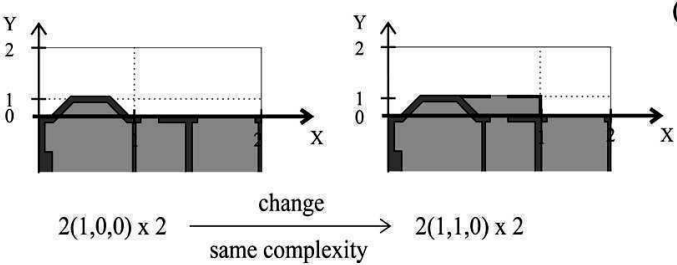

(c)

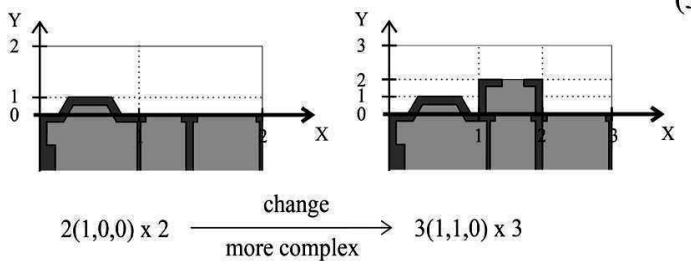

(1)

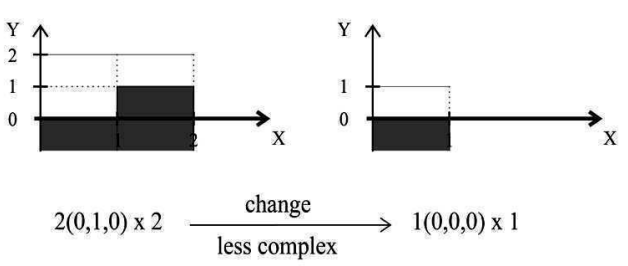

(2)

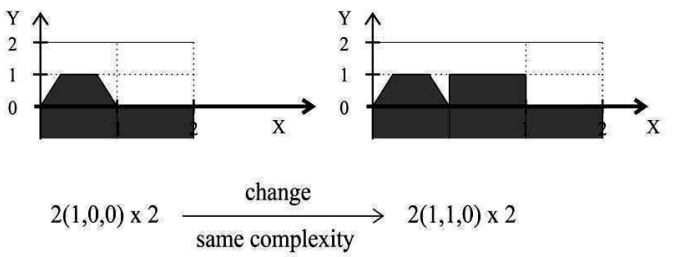

(3)

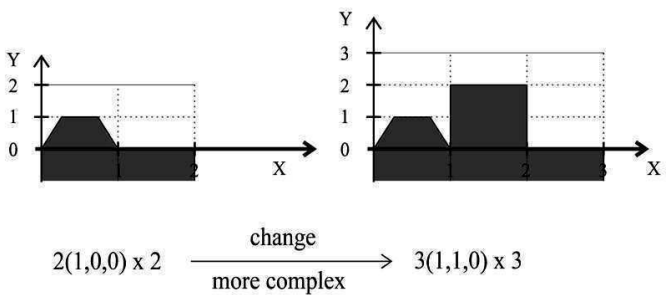

Figure 3.

Three categories of changes to the front interface that either lower the complexity of the interface (a), do not change the complexity as in (b) or raise the complexity as in (c). Diagrams (1), (2) and (3) show the same changes but in dimensionless form (Steadman, 1983). 
Table 1.

The table shows data regarding changes to the front interface. The interface of the semi-detached house seems to have the highest capability to accommodate the change with the highest number of total changes and highest variety of changing types.

\begin{tabular}{|c|c|c|c|}
\hline & Terraced & Semi-detached & Detached \\
\hline \multicolumn{4}{|l|}{ Total number of: } \\
\hline Houses & 2300 & 2258 & 311 \\
\hline Changes to the front interface & 231 & 1433 & 112 \\
\hline Proportion changed & 0.10 & 0.63 & 0.36 \\
\hline \multicolumn{4}{|l|}{ The number of: } \\
\hline Original interface types & 15 & 28 & 14 \\
\hline Original types changed & 11 & 27 & 13 \\
\hline Proportion changed & 0.73 & 0.96 & 0.93 \\
\hline Types that cover $90 \%$ of changes & 4 & 7 & 4 \\
\hline Proportion changed & 0.27 & 0.25 & 0.29 \\
\hline Types that disappeared & 1 & 1 & 0 \\
\hline New types introduced by change & 1 & 8 & 14 \\
\hline Current front interface types & 15 & 35 & 28 \\
\hline \multicolumn{4}{|l|}{ Number of changes that resulted in: } \\
\hline Lower complexity & 82 & 605 & 92 \\
\hline No change to the complexity & 13 & 534 & 8 \\
\hline Higher complexity & 136 & 294 & 12 \\
\hline
\end{tabular}

the space between the house and the street. The reason for this change can be two-fold. The internal layout of the terraced house already provided an entrance hall and having additional space in form of a porch seemed redundant. Moreover, the porches were wooden and proved to be difficult to maintain and prone to damage or decay, which might have been a reason to remove them. In semi-detached housing the division of interface typologies follows the one established in terraced housing. We also see a similar correlation between the type of the change and the original complexity of the interface with the interfaces with medium complexity accommodating the largest number and variety of changes. When the structure of the interface had high complexity (e.g. $3 \times 2,3 \times 3$ ) in 90 per cent of the cases the complexity was lowered, while in the interfaces with low complexity change resulted in higher complexity. The variety of changes was the most numerous in semidetached housing with more than 95 per cent of the changes were achieved by addition to the geometry. The most common change to the semi-detached interface was the addition of a garage in the space to the side of the building. The second most common change was addition of the porch, which might have been motivated by the lack of the entrance hall in the internal layout of the house and the need for space that could mediate the transition from public to private. Even though the complexity categories of the interface fall in line with the terraced and semi-detached examples, the majority of original front interfaces had a high complexity (e.g. $3 \times 3,3 \times 4$ or $3 \times 5$ ). Most of the changes were found to simplify the interface between the house and the street by adding geometry to the existing building. The most common change was the addition of a porch in order to lower the complexity of the threshold. In some cases this change could be motivated by lack of the entrance hall in the internal layout of the house, but there were cases when the entrance hall was part of the layout and it was extended 

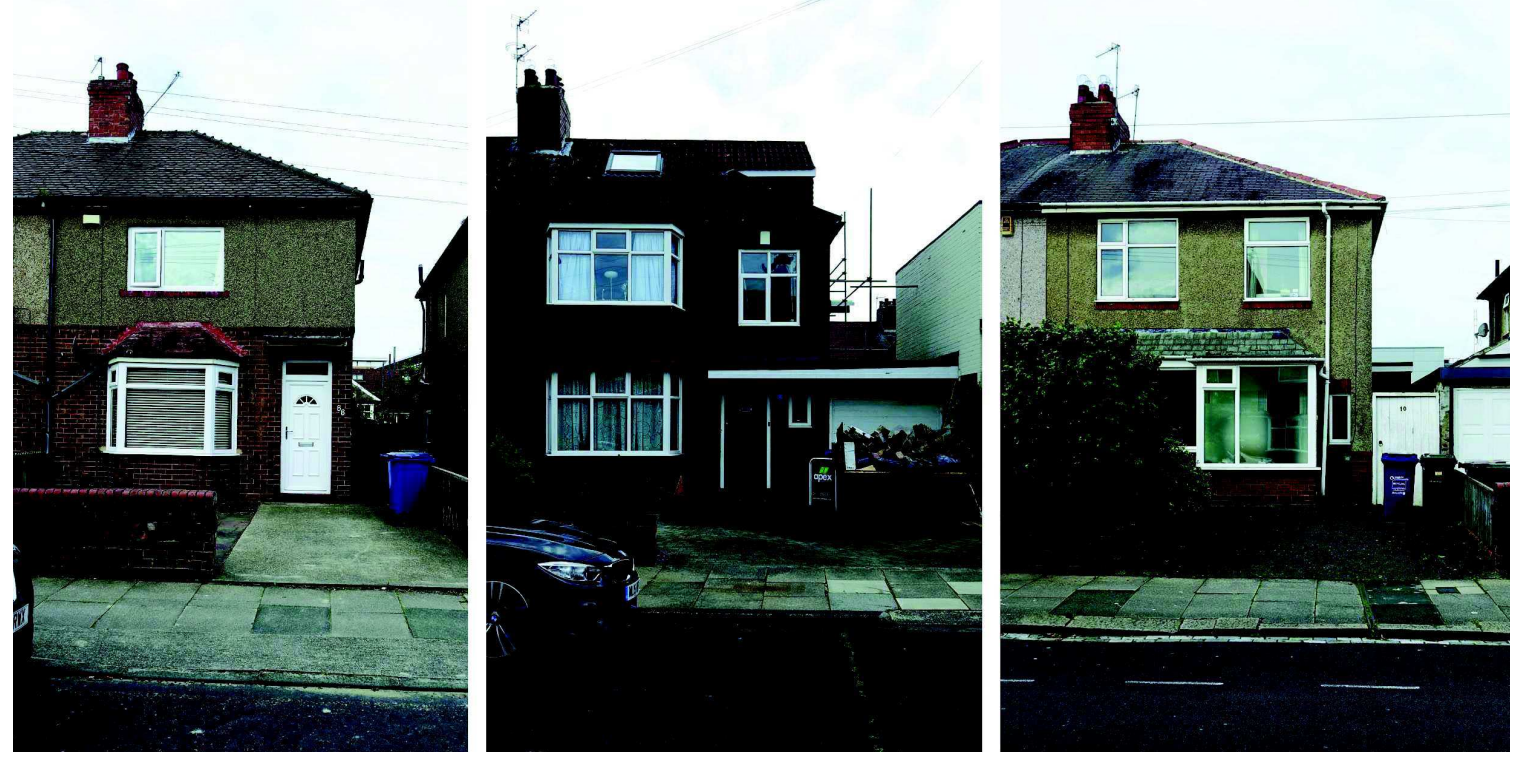

Figure 4.

Examples of incremental changes to the front interface in the Gosforth area. The original typology of a semi-detached house without any changes (on the left). The addition of the garage to the front interface, which did not affect the complexity of the interface plan (in the middle). The addition of the porch to the front interface divided the interface into $3 \times 3$, compared to the $2 \times 2$ in the original example, which introduces more steps to the entrance of the house (on the right).

to the outside to simplify the complex structure of the detached front interface.

\section{Conclusion}

In each interface type, regardless of the housing typology, at least one case was incrementally adjusted by the inhabitants, which shows that each type has the capability to accommodate change if necessary. However, the frequency of changes within those types varies greatly, with some interface types being more likely to change while others rarely changing. The complexity of the original interface type seems to dictate what type of change the interface is capable of accommodating. Interfaces with low complexity (e.g. 1 x 1), when changed, are more likely to increase in complexity, while interfaces with a high complexity (e.g. $3 \times 2$ ), when changed, are more likely to lower in complexity. The interfaces with medium complexity seem to accommodate the largest number and variety of changes and incremental adjustments can lead to all categories of complexity. Thus, the decision made during the design process might determine how adaptable the interface is to accommodate future, unforeseen change. Moreover, the introduction of a space to the side of the building seems to increase the probability of change to the interface and gives the inhabitants a choice of whether to expand or not. The space, however, has to be wide enough to provide enough room for expansion. As seen in detached houses in Gosforth the spaces to the side of the building are very narrow such that any type of expansion is unlikely. Addressing the capability of the building to change and grow during the design process is critical because housing stock does not change often enough. This is visible in the example of terraced housing and the rising popularity of cars. The introduction of the car meant that there was a need to accommodate a place to store it. In the case of terraced houses it could only be stored in the back yard, however narrow access routes - back alleys - were unfit causing residents to park on the street in front of the house. This need introduced a previously unintended environment and changed the function of the existing space. Thus, considering the capability 
to accommodate change in any form, might prove beneficial when there is a need to adjust the designed environment without affecting spaces in a negative way.

\section{Notes}

1 A housing type specific to Newcastle and Tyneside, a terraced house divided into two flats, with one dwelling situated on the ground floor and second dwelling on the first floor.

2 The database available on the Newcastle City Council website - https://www.newcastle. gov.uk/planning-and-buildings/planningapplications/search-and-comment-onplanning-applications. Accessed: 13.06.17.

\section{References}

Altman, I. (1975) The Environment and Social Behavior: privacy, personal space, territory, crowding (Brooks/Cole Pub. Co).

Conzen, M. R. G. (1960). Alnwick, Northumberland: a study in town-plan analysis. Transactions and Papers (Institute of British Geographers) 27, iii-122.

Gehl, J. (1986) 'Soft edges in residential streets'. Scandinavian Housing and Planning Research 3(2), 89-192

Gehl, J. (2013) Cities for People (Island Press, Washington DC).

Habraken, N. J. and Teicher, J. (2000) The structure of the ordinary: form and control in the built environment (MIT press, Cambridge).

Hillier, B. and Hanson, J. (1984) The Social Logic of Space (Cambridge: Cambridge University Press).

Jacobs, J. (1961) The Death and Life of Great American Cities (Middlesex: Penguin, Harmondsworth).

Kropf, K. (2009) 'Aspects of urban form'. Urban Morphology 13(2), 105-120.

Lawrence, R. J. (1987) Housing, dwellings and homes: Design theory, research and practice (John Wiley, Chichester).

Palaiologou, G., Griffiths, S., and Vaughan, L. (2016), 'Reclaiming the virtual community for spatial cultures: Functional generality and cultural specificity at the interface of building and street'. Journal of Space Syntax 7(1), 25-54.

Steadman, P. (1983) Architectural morphology: an introduction to the geometry of building plans (Taylor \& Francis).

Whitehand, J. W. R. and Morton, N. J. and Carr, C. M. H. (1999) 'Urban Morphogenesis at the Microscale: How Houses Change', Environment and Planning B: Planning and Design 26(4), 503-515. 